\title{
Perioperative stroke in a patient undergoing surgery for oral cancer: A case report
}

\author{
SHINTARO SUKEGAWA ${ }^{1}$, TAKAHIRO KANNO ${ }^{2}$, KENGO KANAI $^{3}$, TOSHIKO MANDAI ${ }^{1}$, \\ AKANE SHIBATA $^{1}$, YUKA TAKAHASHI $^{1}$, YUJI HIRATA $^{3}$ and YOSHIHIKO FURUKI $^{3}$ \\ ${ }^{1}$ Division of Oral and Maxillofacial Surgery, Kagawa Prefectural Central Hospital, Takamatsu, Kagawa 760-8557; \\ ${ }^{2}$ Department of Oral and Maxillofacial Surgery, Shimane University Faculty of Medicine, Izumo, Shimane 693-8501; \\ ${ }^{3}$ Department of Otorhinolaryngology, Kagawa Prefectural Central Hospital, Kagawa 760-8557, Japan
}

Received December 13, 2014; Accepted January 19, 2016

DOI: $10.3892 / \mathrm{ol} .2016 .5031$

\begin{abstract}
Carotid artery stenosis is a significant risk factor for stroke. In elderly patients with carotid atherosclerosis and stenosis, it is not unusual for oral, head and neck cancer surgery to be performed. The present study describes a case of stroke that occurred during a neck dissection for the treatment of cervical lymph node metastasis of a left maxillary gingival carcinoma. The patient was an 84-year-old female who was considered to be at high risk of a stroke based on pre-operative head and neck computed tomography scans, which detected severe carotid atherosclerosis and stenosis. There was no possible stroke prophylaxis available during the performance of the neck dissection in the present case. However, if patients are evaluated to be high-risk pre-operatively, statin agents should be administered, the surgery should be carefully performed, adequate sedation should be maintained post-operatively and the patient should be followed up, aiming to achieve the early detection of a possible stroke.
\end{abstract}

\section{Introduction}

In contrast to patients with numerous other carcinomas in which fatality most often occurs as a result of disseminated malignant disease, patients with oral, head and neck carcinoma typically succumb to the disease with a lack of clinical evidence of metastasis beyond regional or local disease (1). Therefore, use of a neck dissection is important to control regional spread to the lymph nodes and to prevent localized spread (2). Neck dissection involves the neurological and vascular structures of the neck being exposed and manipulated, which may lead to the complication of perioperative stroke. The occurrence of

Correspondence to: Dr Shintaro Sukegawa, Division of Oral and Maxillofacial Surgery, Kagawa Prefectural Central Hospital, 1-2-1 Asahi-cho, Takamatsu, Kagawa 760-8557, Japan

E-mail: gouwan19@gmail.com

Key words: carotid atherosclerosis, neck dissection, perioperative complication, oral squamous cell carcinoma, stroke perioperative stroke during neck and head surgery is a severe complication associated with a high risk of morbidity and mortality. Nosan et al (3) and Thompson et al (2) reported that the incidence rates of perioperative stroke in patients with head and neck cancers were 4.8 and $0.2 \%$, respectively.

Perioperative stroke is a cerebrovascular incident that can arise intraoperatively (4). Carotid artery stenosis is a significant risk factor for stroke, and is associated with various other factors, including smoking, peripheral vascular disease, hypertension, diabetes, male gender, atherosclerotic heart disease, age and external radiotherapy to the head and neck (5-7). Patients with head and neck cancer undergoing a neck dissection may demonstrate a number of these risk factors, therefore predisposing them to perioperative stroke.

The present study describes the case of a female patient who experienced perioperative stroke during surgery for the treatment of oral, head and neck cancer.

\section{Case report}

In August 2013, an 84-year-old female was referred to a general dental practitioner for gingival swelling and pain in the upper left molar region. A red, painful ulcer was noted during the examination and the patient was subsequently referred to the Division of Oral and Maxillofacial Surgery, Kagawa Prefectural Central Hospital (Takamatsu, Japan) for evaluation of a possible maxillary gingival carcinoma also in August 2013. Extraoral examination detected slight left-sided facial asymmetry and tenderness. The upper jugular and left submandibular lymph nodes were palpable and tender. Intraoral examination detected a $25 \times 35-\mathrm{mm}$ mass in the palatal and buccal aspect of the left maxillary edentulous alveolus, in the proximity of the first premolar and the second molar. The mucosal surface of the mass was rough and tender, and was covered with numerous pink-red, pebbly, hemorrhagic papules that bled on palpation. The medical history of the patient included hypertension and hyperlipidemia, for which the patient was taking an angiotensin II receptor antagonist (losartan potassium), and a calcium channel blocker (amlodipine) and a 3-hydroxy-3-methylglutaryl-coenzyme A reductase inhibitor (statin; pravastatin sodium), respectively. There was no history of cerebral infarction. 

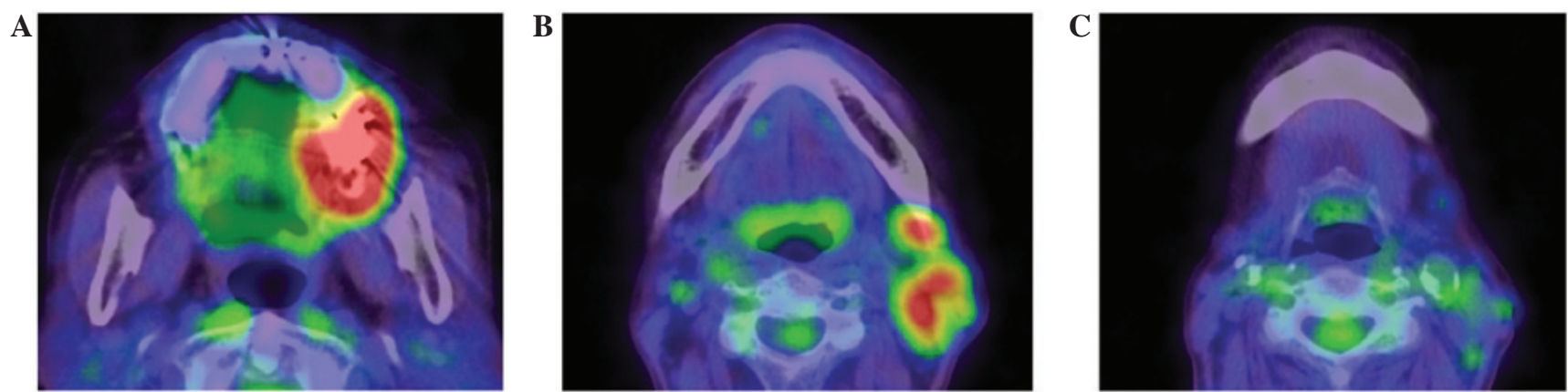

Figure 1. ${ }^{18} \mathrm{~F}$-fluorodeoxyglucose-positron emission tomography demonstrating increased uptake in the (A) left maxilla (SUVmax, 10.5) and (B) multiple regional lymph nodes (SUVmax, 5.0), (C) but no active uptake in the calcified carotid artery. SUVmax, maximum standardized uptake value.

Computed tomography (CT; Aquilion 64; Toshiba America Medical Systems, Inc., Tustin, CA, USA) revealed a 42x40-cm, enhanced lesion, which extended from the left maxillary alveolar region to the maxillary sinus, buccal mucosa and hard palate, in addition to a number of enhanced lymph nodes in the ipsilateral neck. ${ }^{18} \mathrm{~F}$-fluorodeoxyglucose-positron emission tomography (FDG-PET; Biograph mCT40; Siemens Healthcare, Erlangen, Germany) detected increased uptake in the left maxilla [maximum standardized uptake value (SUVmax), 10.5; Fig. 1A] and multiple regional lymph nodes (SUVmax, 5.0; Fig.1B). ${ }^{18}$ F-FDG-PET did not demonstrate active uptake in the calcified carotid artery (Fig. C), and no abnormal uptake suggestive of second primary or distant metastasis was detected.

From these observations, the tumor was clinically staged as T4aN2bM0 in accordance with the 2010 Union for International Cancer Control system (8). An incisional biopsy was performed and following inspection (hematoxylin and eosin staining), during which squamous differentiation was observed in the form of keratinization with variable pearl formation, loss of the basement membrane and disturbed architecture of the basal layers of the epithelium, the lesion was diagnosed as squamous cell carcinoma (SqCC). Thus, a left subtotal maxillectomy with a left neck dissection was planned. Pre-operative CT revealed an advanced, calcified plaque in the contralateral right carotid artery that required neck dissection (Fig. 2); therefore, the surgery was performed without discontinuing the statin treatment that the patient had been administered for several years. In September 2013, a left subtotal maxillectomy, left neck dissection and tracheotomy were performed under general anesthesia (Fig. 3).

Following the surgery, the patient was transferred to the intensive care unit (ICU) of the Kagawa Prefectural Central Hospital. The respiratory and circulatory systems were managed under sedation. The patient was maintained on mechanical ventilation under continuous dexmedetomidine (DEX; 0.2-0.7 $\mu \mathrm{g} / \mathrm{kg} / \mathrm{h}$ ) sedation. The level of sedation was reduced once the patient achieved spontaneous, stable breathing, which occurred at $6 \mathrm{~h}$ post-surgery. At that time, the patient was poorly responsive, with no movement of the right-sided extremities. Brain magnetic resonance (MR) imaging (Magnetom Avanto; Siemens Healthcare) revealed a fresh watershed area infarction in the left middle cerebral artery (Fig. 4), and MR angiography exhibited no obvious vascular stenosis. Edaravone $(60 \mathrm{mg})$ was administered twice

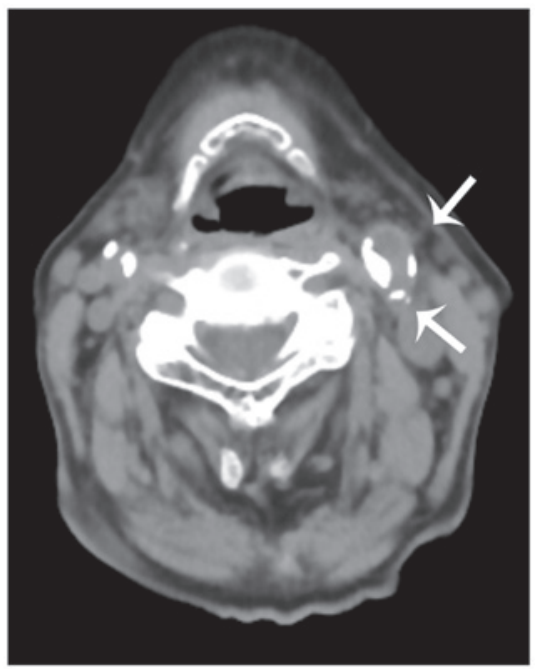

Figure 2. Pre-operative computed tomography revealing advanced, calcified stenosis (arrows) in the contralateral right carotid artery requiring neck dissection.
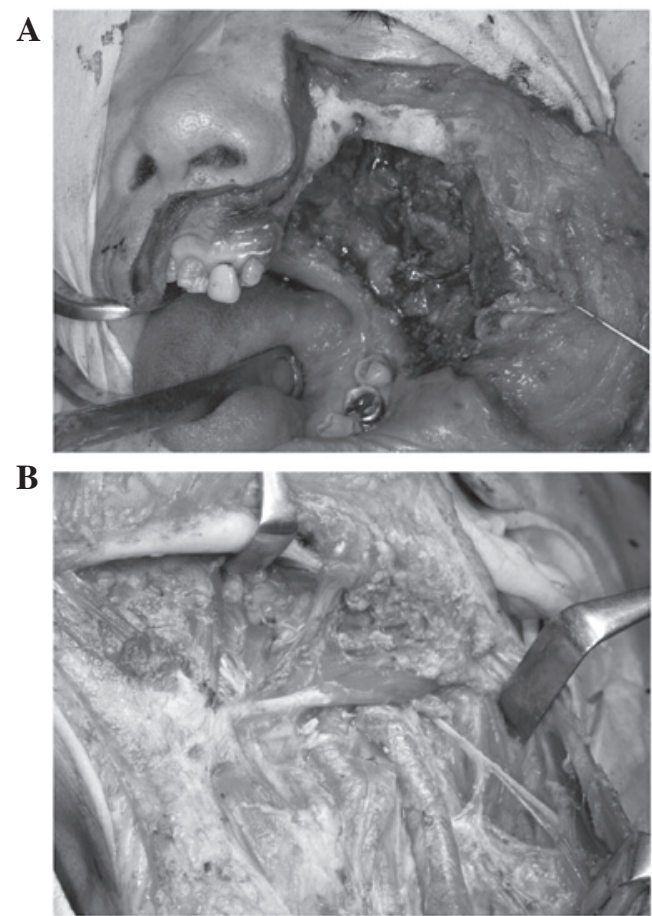

Figure 3. Images captured from the (A) left subtotal maxillectomy and (B) left neck dissection, showing no notable intraoperative complications or abnormalities. 

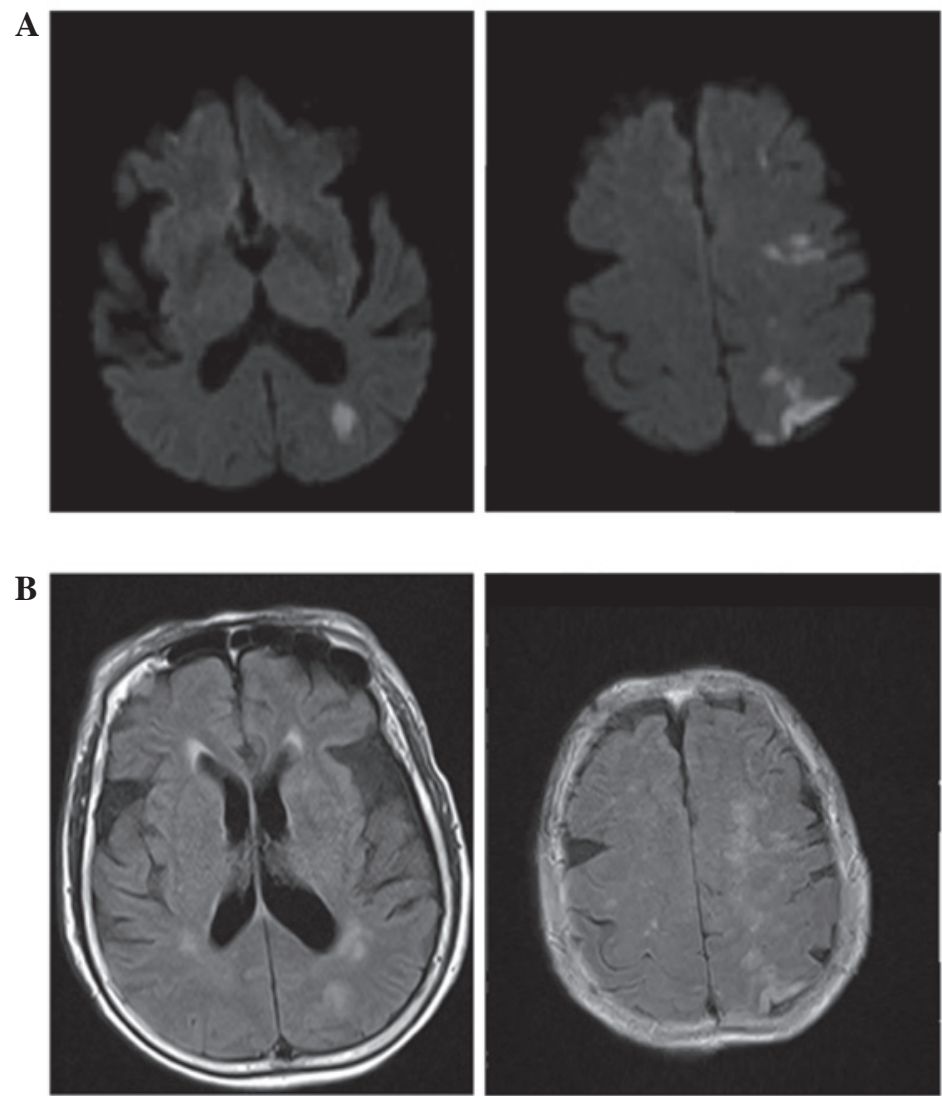

Figure 4. Magnetic resonance imaging exhibiting a fresh watershed area with punctiform, high-signal, multiple infarctions in the left middle cerebral artery. (A) Diffusion-weighted images. (B) Fluid-attenuated inversion recovery images.

per day for 2 days, and continuous respiratory and circulatory management was performed. On day 2 post-surgery, the patient began rehabilitation in the ICU. As there were no complications of the respiratory and circulatory systems or the surgical site, the patient was discharged from the ICU on day 5 post-surgery. Several days later, the patient demonstrated progressive improvement. Occupational and physical therapists were consulted to evaluate and plan rehabilitation. In order to encourage mobility using a caster walker, the patient was discharged on day 38 post-surgery. At the 6-month post-operative follow-up, the patient was able to walk using a walking stick and perform normal daily tasks with minimal residual weakness. Approximately 1 year subsequent to surgery, the patient exhibited recurrence from the primary tumor, and underwent radiation treatment at Takinomiya General Hospital (Ayauta, Japan). The patient succumbed to disease due to airway obstruction in June 2015.

\section{Discussion}

Lymph node metastases occur in $>50 \%$ of patients with oral $\mathrm{SqCC}$, and histological confirmation of metastatic disease is a key prognostic factor (9). Localized spread may be prevented by tumor excision and neck dissection, which aim to control the regional spread to the lymph nodes (2). In contrast to non-neck procedures, neck dissections carry a high risk of cerebrovascular accidents; this is primarily due to the neck often being rotated and hyperextended during surgery, which may result in plaque ulceration from turbulent flow or intimal tearing of the carotid artery and thrombus formation. In addition, the carotid artery is frequently retracted, which may dislodge a thrombus or plaque (10).

The incidence of perioperative stroke during the performance of non-neck and head surgery is $0.08-0.2 \%$ (11). There are a limited number of reports regarding the incidence of perioperative stroke in patients who undergo neck dissection. Nosan et al (3) retrospectively reviewed 5 patients that had undergone neck dissection and reported a $4.8 \%$ incidence of perioperative stroke occurring during surgery. A study by Rechtweg et al (11) described 3 cases of simultaneous carotid endarterectomy and neck dissection. In the discussion section, the study reported an unpublished personal communication from Yoo et al (unpublished data), who reviewed 441 patients undergoing neck dissections in Toronto, Canada, and found an incidence of perioperative stroke of $3.2 \%$. By contrast, Thompson et al (2) reported that the incidence of perioperative stroke in 499 patients undergoing a neck dissection for cancer of the neck and head was as low as $0.2 \%$. However, the data from Thompson et al (2) was obtained from a historical cohort that analyzed hospital discharge data, and it may be possible that data-entry errors occurred. Further large-scale clinical studies are required to confirm these incidence rates.

Carotid artery stenosis is the most significant risk factor for stroke (12). Atherosclerotic plaque vulnerability represents a crucial target to decrease the probability of plaque rupture and the thrombotic ischemic events that follow $(13,14)$. CT/FDG-PET facilitates highly precise measurements of the inflammatory activity of atherosclerotic plaques $(15,16)$. 
The patient of the present case presented with a number of risk factors, including a calcification in the carotid artery revealed by CT, although an unstable plaque did not exist on FDG-PET/CT. Therefore, surgery was performed without discontinuing the statin treatment that the patient had been administered for several years. Local and systemic inflammatory mediators favor the increase of plaque vulnerability. Treatment with statins has been demonstrated to diminish inflammatory processes and increase collagen stabilization in carotid atherosclerotic plaques (17). Furthermore, it has been reported that treatment with statins leads to decreased macrophage accumulation in the carotid plaques of patients with hypercholesterolemia and significant carotid stenosis when compared with cholestyramine plus $\beta$-sitosterol treatment (18).

Head and neck surgeries can often be prolonged; thus, patients may require post-operative sedation (19). If it is determined that there is a high risk of post-operative stroke, neurological assessments are required while the patient is sedated. Traditional agents, including propofol, lorazepam and midazolam, induce sedation by acting on $\gamma$-aminobutyric acid receptors; therefore, it is difficult to judge the neurological state of the patient when treated with such agents. By contrast, DEX, a selective $\alpha 2$-adrenergic receptor agonist, sustains a natural sleep pattern and evokes cooperative sedation from which patients are easily aroused. In addition, DEX results in reduced impairment of post-operative cognitive function (20); thus, DEX allows patients to awaken for neurological evaluation.

In conclusion, the present case demonstrates that perioperative stroke can occur in patients undergoing surgery for oral, neck and head cancer. In the current case, there was no prophylaxis for the occurrence of stroke during the neck dissection. However, if patients are pre-operatively judged to be at high risk, pre-operative statins should be administered, the surgery should be carefully performed, adequate sedation should be maintained post-operatively and the patient should be cautiously observed for the early detection of stroke.

\section{References}

1. Coleman JJ and Sultan MR: Tumors of the head and neck. In: Principles of Surgery. 7th edition. Schwartz SI, Shires GT, Spencer FC, Daly JM, Fischer JE and Galloway AC (eds). McGraw-Hill, New York, pp601-665, 1999.

2. Thompson SK, Southern DA, McKinnon JG, Dort JC and Ghali WA: Incidence of perioperative stroke after neck dissection for head and neck cancer: A regional outcome analysis. Ann Surg 239: 428-431, 2004.

3. Nosan DK, Gomez CR and Maves MD: Perioperative stroke in patients undergoing head and neck surgery. Ann Otol Rhinol Laryngol 102: 717-723, 1993.
4. Kim J and Gelb AW: Predicting perioperative stroke. J Neurosurg Anesthesiol 7: 211-215, 1995.

5. Bashar K, Healy D, Clarke-Moloney M, Burke P, Kavanagh E and Walsh SR: Effects of neck radiation therapy on extra-cranial carotid arteries atherosclerosis disease prevalence: Systematic review and a meta-analysis. PLoS One 9: e110389, 2014.

6. Lam WW, Yuen HY, Wong KS, Leung SF, Liu KH and Metreweli C: Clinically underdetected asymptomatic and symptomatic carotid stenosis as a late complication of radiotherapy in Chinese nasopharyngeal carcinoma patients. Head Neck 23: 780-784, 2001.

7. Cheng SW, Wu LL, Ting AC, Lau H, Lam LK and Wei WI: Irradiation-induced extracranial carotid stenosis in patients with head and neck malignancies. Am J Surg 178: 323-328, 1999.

8. Edge S, Byrd DR, Compton CC, Fritz AG, Greene FL and Trotti A (eds): AJCC Cancer Staging Manual. 7th edition. Springer, Chicago, IL, USA, 2010.

9. Kowalski LP and Sanabria A: Elective neck dissection in oral carcinoma: A critical review of the evidence. Acta Otorhinolaryngol Ital 27: 113-117, 2007.

10. Atik MA, Ates M, Akkus NI, Altundag $\mathrm{O}$ and Altundag K: Preoperative Doppler sonography for prevention of perioperative stroke in head and neck cancer patients undergoing neck dissection: Is it beneficial? J Clin Ultrasound 35: 38-39, 2007.

11. Rechtweg J, Wax MK, Shah R, Granke K and Jarmuz T: Neck dissection with simultaneous carotid endarterectomy. Laryngoscope 108: 1150-1153, 1998.

12. Calliada F, Verga L, Pozza S, Bottinelli O and Campani R: Selection of patients for carotid endarterectomy: The role of ultrasound. J Comput Assist Tomogr 23 (Suppl 1): S75-S81, 1999.

13. Naghavi M, Libby P, Falk E, Casscells SW, Litovsky S, Rumberger J, Badimon JJ, Stefanadis C, Moreno P, Pasterkamp G, et al: From vulnerable plaque to vulnerable patient: A call for new definitions and risk assessment strategies: Part I. Circulation 108: 1664-1672, 2003

14. Naghavi M, Libby P, Falk E, Casscells SW, Litovsky S, Rumberger J, Badimon JJ, Stefanadis C, Moreno P, Pasterkamp G, et al: From vulnerable plaque to vulnerable patient: A call for new definitions and risk assessment strategies Part II. Circulation 108: 1772-1778, 2003

15. Mehta NN, Yu Y, Saboury B, Foroughi N, Krishnamoorthy P, Raper A, Baer A, Antigua J, Van Voorhees AS, Torigian DA, et al: Systemic and vascular inflammation in patients with moderate to severe psoriasis as measured by $[18 \mathrm{~F}]$-fluorodeoxyglucose positron emission tomography-computed tomography (FDG-PET/CT): A pilot study. Arch Dermatol 147: 1031-1039, 2011.

16. Yun M, Yeh D, Araujo LI, Jang S, Newberg A and Alavi A: F-18 FDG uptake in the large arteries: A new observation. Clin Nucl Med 26: 314-319, 2001.

17. Crisby M, Nordin-Fredriksson G, Shah PK, Yano J, Zhu J and Nilsson J: Pravastatin treatment increases collagen content and decreases lipid content, inflammation, metalloproteinases and cell death in human carotid plaques: Implications for plaque stabilization. Circulation 103: 926-933, 2001.

18. Puato M, Faggin E, Rattazzi M, Zambon A, Cipollone F, Grego F, Ganassin L, Plebani M, Mezzetti A and Pauletto P: Atorvastatin reduces macrophage accumulation in atherosclerotic plaques: A comparison of a nonstatin-based regimen in patients undergoing carotid endarterectomy. Stroke 41: 1163-1168, 2010.

19. Paul BS and Paul G: Sedation in neurological intensive care unit. Ann Indian Acad Neurol 16:194-202, 2013.

20. Yu SB: Dexmedetomidine sedation in ICU. Korean J Anesthesiol 62: 405-411, 2012. 